\title{
Effects of higher dimension operators on the Standard Model Higgs sector
}

\author{
Oscar Åkerlund* \\ Institut für Theoretische Physik, ETH Zurich, CH-8093 Zürich, Switzerland \\ E-mail: oscara@itp.phys.ethz.ch
}

\section{Philippe de Forcrand}

Institut für Theoretische Physik, ETH Zurich, CH-8093 Zürich, Switzerland CERN, Physics Department, TH Unit, CH-1211 Geneva 23, Switzerland

E-mail: forcrandeitp.phys.ethz.ch

\section{Jakob Steinbauer}

Institut für Theoretische Physik, ETH Zurich, CH-8093 Zürich, Switzerland

E-mail: jakobstedstudent.ethz.ch

\begin{abstract}
We study the effect of higher dimension operators on the electroweak finite temperature phase transition in two sectors of the Standard Model. Firstly, the Higgs-Yukawa sector, consisting of the Higgs doublet and the massive Standard Model fermions, is studied with an approximate method, Extended Mean Field Theory. Secondly, the gauge-Higgs sector, consisting of the Higgs doublet and the gauge fields of the weak interaction, is studied using Monte Carlo simulations. In both cases we find that a cutoff scale of around $1.5 \mathrm{TeV}$ is needed to make the electroweak phase transition first order at the experimental value of the Higgs boson mass, which is a requirement for making electroweak baryogenesis viable.
\end{abstract}

The 33rd International Symposium on Lattice Field Theory

14 -18 July 2015

Kobe International Conference Center, Kobe, Japan

${ }^{*}$ Speaker. 


\section{Introduction}

Despite its outstanding success, the Standard Model (SM) is incomplete ${ }^{1}$ and can thus only be considered an effective model. Due to its renormalizability, and the (seemingly) special value of the Higgs boson mass, however, its range of validity seems to be huge and it may even be internally consistent all the way up to the Planck scale [1,2]. This is both intriguing and challenging, since any UV completion of the SM must leave most of its observables almost unchanged at the same time as it solves the problems of the SM, such as dark matter, neutrino oscillations and baryogenesis. Yet, although the SM does not require new physics to be important until very high energies, there is no general reason to exclude new degrees of freedom with masses around a few TeV. Something similar happens in the Fermi Theory of Weak interactions where the mass of the $W$-boson of $80 \mathrm{GeV}$ is significantly below $4 \pi\langle\phi\rangle \approx 3 \mathrm{TeV}$, where the theory stops being perturbative.

In this proceeding we study generic effects of Beyond the Standard Model (BSM) physics at intermediate scales by including the dimension six operator $|\phi|^{6}$ in the SM Higgs sector. Generally, there are of course many more higher order operators [3] but for simplicity we will let $|\phi|^{6}$ serve as a proxy for all of them. The main focus will be on the order of the electroweak finite temperature transition as a function of the Higgs mass $M_{h}$ and of the coefficient of the dimension six operator, parametrically given by $M_{\mathrm{BSM}}^{-2}$, where $M_{\mathrm{BSM}}$ can be thought of as the mass of the lightest particle in the UV completion of the SM which couples to the Higgs boson. The motivation behind this is to investigate the possibility of electroweak baryogenesis, which, due to one of the three Sakharov conditions for baryogenesis, can only be viable if there is a first order transition in the electroweak sector as the universe cools down. With no dimension six operator, the transition is a crossover for the Higgs mass at its experimental value $125 \mathrm{GeV}$, and it would only become first order for a Higgs mass below about $70 \mathrm{GeV}$ [4]. However, in the presence of a $|\phi|^{6}$ operator the critical value of the Higgs mass can be raised and it is interesting to determine for which value of $M_{\mathrm{BSM}}$ the critical Higgs mass exceeds the experimental value. Since it is not known how to represent the full SM on the lattice we answer this question in two different simplified versions of it: first in the Higgs-Yukawa model which neglects all gauge fields but retains the Higgs field and all (massive) SM fermions, and then in a gauge-Higgs model consisting of the Higgs field and the $S U(2)$ gauge fields.

\section{Higgs-Yukawa model}

The Higgs-Yukawa model neglects the gauge degrees of freedom of the SM and consists of the complex, two component, scalar Higgs field and the massive SM fermions, coupled to the Higgs field via Yukawa interaction terms. Since, at tree level, the Yukawa couplings $y_{f}=M_{f} /\langle\phi\rangle$ are proportional to the masses of the fermions $M_{f}$, and since the top quark mass $M_{t}$ is of the same order of magnitude as the Higgs expectation value $\langle\phi\rangle$, it is important to treat the model nonperturbatively. Due to the chiral nature of the Yukawa coupling it is also important to use a chiral Dirac operator on the lattice, for example the Neuberger Overlap operator. This in turn makes full, dynamical lattice simulations of the model very demanding and expensive in terms of computer time. We will follow a different route and solve the model approximately using Extended Mean

\footnotetext{
${ }^{1}$ Most obviously due to the fact that it is completely neglecting gravity.
} 
Field Theory (EMFT) which comprises a self-consistent determination of the Higgs expectation value and self-energy due to the self- and Yukawa-interactions. In this approximation the fermions couple to the magnitude $\left|\varphi_{0}\right|$ of the Higgs field, which is taken to be constant in space time so that the Dirac operator $D^{\text {ov }}$ factorizes into independent blocks, one for each fermion. The magnitude varies, however, as we integrate over all the components of the Higgs field. The lattice action takes the form

$$
\begin{aligned}
S=\sum_{x}\{ & -\kappa \sum_{\mu} \varphi_{x}^{\dagger} \varphi_{x+\hat{\mu}}+\text { h.c. }+\left|\varphi_{x}\right|^{2}+4 \kappa^{2} \lambda\left(\left|\varphi_{x}\right|^{2}-1\right)^{2}+8 \kappa^{3} \lambda_{6}|\varphi|^{6} \\
& \left.+\sum_{f} N_{c, f} \operatorname{Tr} \log \left(D^{\mathrm{ov}}\left(y_{f} \sqrt{2 \kappa}\left|\varphi_{0}\right|\right)\right)\right\},
\end{aligned}
$$

where $\kappa$ is inversely proportional to the bare mass squared and where $\lambda_{6}=\left(a M_{\mathrm{BSM}}\right)^{-2} \cdot N_{c, f}$ is a multiplicity factor which equals 3 for quarks (number of colors) and 1 for leptons.

This action is then solved using EMFT, see [5] for details, and in particular we determine the finite temperature phase diagram in the $\left(M_{\mathrm{BSM}}, M_{h}\right)$-plane. As a consistency check, we first compare the EMFT solution to full Monte Carlo simulations [6, 7], which have been performed using a restricted version of the model with only the top and bottom quarks, which were further taken to be mass degenerate (otherwise there is a sign problem), and unit multiplicity factors $N_{c, f}$. In Fig. 1 we show the Higgs expectation value $\langle\phi\rangle$ as a function of the hopping parameter $\kappa$ for various values of the quartic coupling $\lambda$ at a small value of $\lambda_{6}=0.1$ (left panel) and at a larger, nonperturbative value of $\lambda_{6}=1$ (right panel). The symbols are Monte Carlo data and the dashed lines are obtained by a perturbative effective model called the Constraint Effective Potential, both taken from [6, 7]. The solid lines are the EMFT results from this work. Clearly EMFT is an excellent approximation at all parameter values, in addition to being orders of magnitude cheaper computationally than the Monte Carlo simulations.
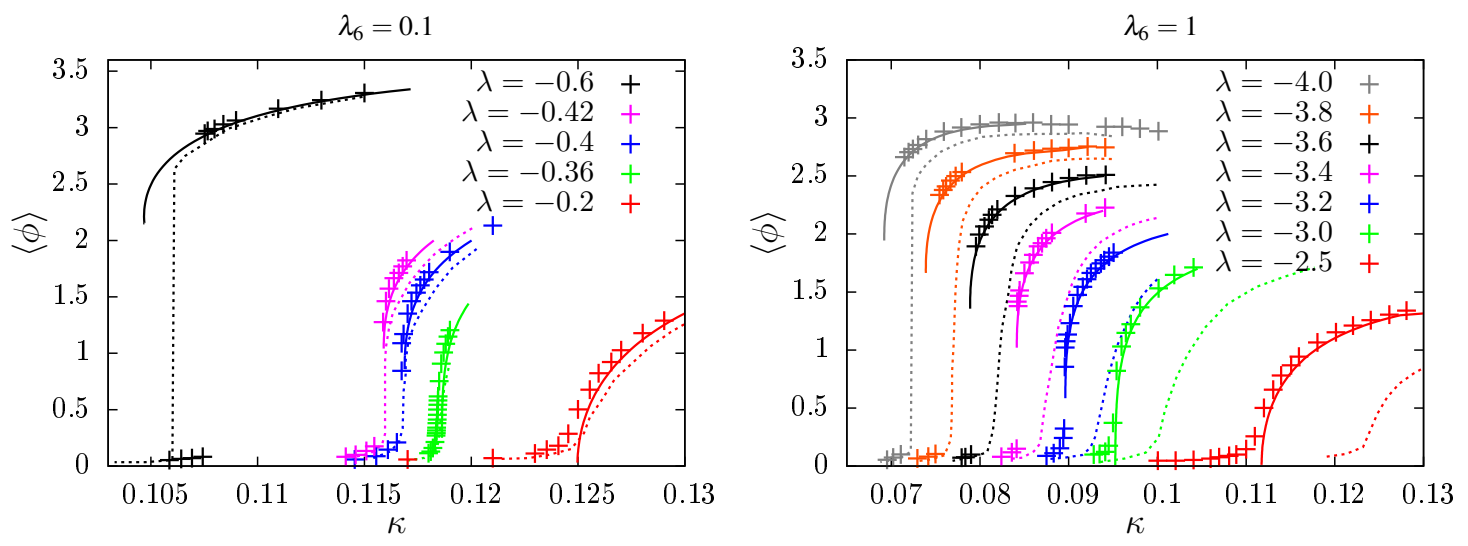

Figure 1: The Higgs expectation value $\langle\phi\rangle$ in lattice units for various quartic couplings $\lambda$ with $\lambda_{6}=0.1$ (left panel) and $\lambda_{6}=1$ (right panel). The symbols and dashed lines are Monte Carlo data and Constraint Effective Potential data respectively, taken from [6, 7]. The solid lines are the EMFT results obtained in this work.

In order to obtain the full phase diagram in physical parameters, we first determine the order of the $\kappa$-driven transition in the $\left(\lambda, \lambda_{6}\right)$-plane at zero temperature. Without gauge fields the expec- 
tation value of the Higgs field is a true order parameter so the transition can be either of first or second order as seen in Fig. 1. For any positive value of $\lambda_{6}$, there is a tricritical value of $\lambda<0$ for which the transition turns from second to first order as $\lambda$ is taken to be more negative. How this tricritical coupling depends on $\lambda_{6}$ can be seen in the left panel of Fig. 2. In the right panel we have fixed $\lambda_{6}=1 / 4$ and we show the phase diagram in the $(\lambda, \kappa)$-plane. The star denotes the tricritical point and corresponds to the intersection of the line in the right panel and the line in the left panel of Fig. 2. We now turn on temperature by making the lattice extent finite in the temporal direction and study the effect on the tricritical point. Its trajectory as the temperature is increased is indicated by the arrow in the right panel of Fig. 2 and since it moves into the region of the symmetry broken phase, we conclude that the symmetry restored phase is expanding, as we know it should since at very high temperature the model is always in the symmetric phase. The interesting region in this phase diagram is the shaded gray area between the red first-order line and the trajectory of the tricritical point. This area is swept out by the first order line as the temperature rises and thus, if the parameters are such that at zero temperature we are in the broken phase somewhere in the gray area, for some finite temperature the first-order line will traverse this point and we will go to the symmetric phase via a first-order phase transition. This is exactly the scenario we are looking for, except in reverse as the universe cools down.
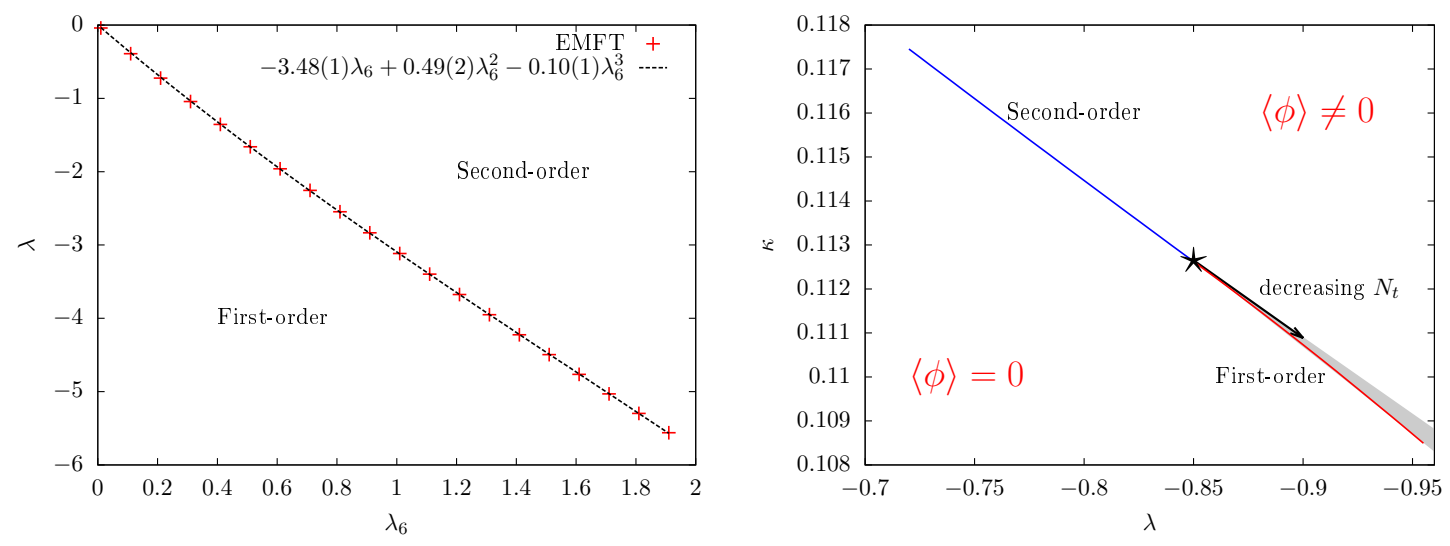

Figure 2: Left panel: The tricritical line at zero temperature in the $\left(\lambda_{6}, \lambda\right)$-plane. For $\lambda$ below the line the transition is first order and above it is second order. Right panel: The phase diagram in the $(\lambda, \kappa)$-plane for $\lambda_{6}=1 / 4$ at zero temperature. On the blue line the transition is second order and on the red line it is first order. The star in between is the tricritical point. As the temperature is increased the tricritical point moves along the black arrow and the first order line sweeps out the gray area which is where a first order finite temperature transition can be found.

The last step consists of considering curves of fixed $\phi_{c} / T_{c}$ inside the gray area, i.e. curves where the expectation value of the field $\phi_{c}$ at the critical temperature $T_{c}$ is constant in units of the critical temperature. A value of 0 corresponds to the trajectory of the tricritical point itself and a value of 1 is generally required in order to have a strong enough first-order transition for electroweak baryogenesis to be viable. In the left panel of Fig. 3 we show this curve in the $\left(M_{\mathrm{BSM}}, M_{h}\right)$ plane for three different values of $\phi_{c} / T_{c}$. The color coding denotes $T_{c}$ in $\mathrm{GeV}$ and the dashed lines show the experimental value of the Higgs mass $M_{h}$ and a nominal value for $M_{\mathrm{BSM}}$. We have fixed the lattice spacing such that $a M_{\mathrm{BSM}}=2$. In the right panel of Fig. 3 we show the impact the 
fermions have on the result by comparing the trajectory of the tricritical point using all nine massive SM fermions and that with only the pure Higgs sector. We do this for two different values of the lattice spacing in terms of $M_{\mathrm{BSM}}$ and find that the effect of fermions is rather small and of indefinite sign, indicating that the physics is dominantly determined by the Higgs self-interaction.
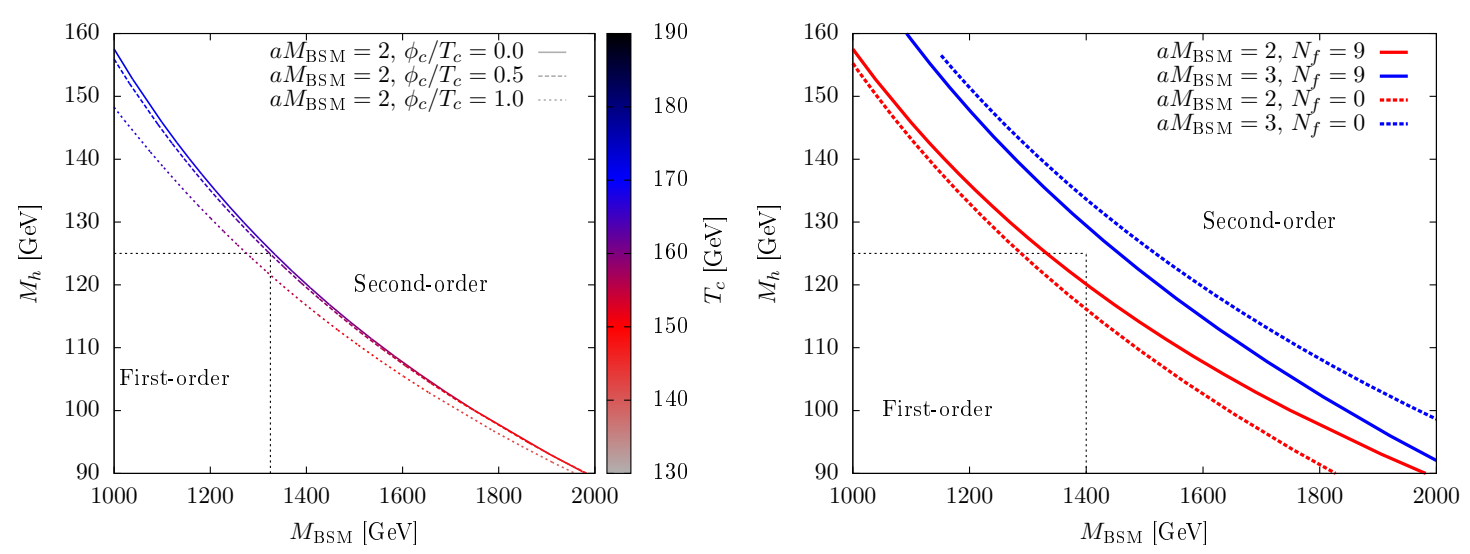

Figure 3: Left panel: The phase diagram in the $\left(M_{\mathrm{BSM}}, M_{h}\right)$-plane. The lines are lines of constant $\phi_{c} / T_{c}$ where $\phi_{c}$ is the expectation value at $T_{c}$. The solid line where $\phi_{c} / T_{c}=0$ is the tricritical line. As $M_{\mathrm{BSM}}$ is decreased the transition becomes stronger. The color coding gives $T_{c}$ in GeV. Right panel: The tricritical line with and without all massive SM fermions at two different values of $a M_{\mathrm{BSM}}$. The spread of these lines gives an estimate of the systematic uncertainties of the effective model. The effect of fermions is rather small.

\section{Gauge-Higgs model}

The gauge-Higgs model consists of the Higgs field and the weak gauge degrees of freedom and thus neglects all fermionic fields. In the light of the result above this seems well justified. Moreover, it is an interesting model on its own, where the gauge fields yield important contributions to the couplings in the thermal effective theory of the Higgs sector [8]. The lattice action for this model is given by

$$
\mathscr{L}_{\mathrm{GH}}=-\sum_{\mu} \kappa_{\mu} \phi^{\dagger}(x) U_{\mu}(x) \phi(x+\hat{\mu})+|\phi(x)|^{2}+\lambda\left(|\phi(x)|^{2}-1\right)^{2}-\frac{1}{2} \sum_{\mu>v} \beta_{\mu v} \operatorname{Re} \operatorname{Tr} P_{\mu v}(x),
$$

where $\kappa_{\mu}$ and $\beta_{\mu v}$ are the anisotropic hopping parameter and gauge coupling respectively. These will take different values for the spatial, $\mu \in\{1,2,3\}$, and temporal, $\mu=4$, directions and we define the bare anisotropy factors to be $\gamma_{\kappa}^{2}=\kappa_{4} / \kappa_{i}$ and $\gamma_{\beta}^{2}=\beta_{4 i} / \beta_{i j}$. The reason for choosing anisotropic couplings is that this will allow us to reach higher temperatures without reducing the spatial lattice size too much and thus the total number of lattice sites can be kept under control. A more complete description of the model and technical details of the simulations can be found in [9] and [10].

At tree level the bare anisotropy factors should be equal since they both represent the ratio $\gamma_{\kappa}=\gamma_{\beta}=a_{s} / a_{t}$ of lattice spacings in the spatial and temporal directions. However, due to quantum effects they get renormalized and acquire different corrections. So in order to have a renormalized anisotropy $\xi=a_{s} / a_{t}$ different from one, $\gamma_{K}$ and $\gamma_{\beta}$ need to be tuned individually. In the Higgs channel we use the ratio of spatial versus temporal Higgs masses to tune $\gamma_{\kappa}$ and in the gauge 
channel we use ratios of elongated Wilson loops in spatial versus temporal planes to tune $\gamma_{\beta}$. For the parameter values we considered they both obtain small corrections, not too different from 1loop perturbative values, which however depend rather strongly on the exact values of the bare couplings.

After the anisotropies have been tuned at zero temperature, we can determine the phase diagram in the $(\lambda, \kappa)$-plane at finite temperature, which is shown in the left panel of Fig. 4 where a lattice of size $20^{3} \times 2$ has been used. It is known that the Higgs mass is increasing with $\lambda$, so we expect that for small $\lambda$ the transition will be first order and for large $\lambda$ it will be a crossover. Somewhere in between there is a critical value where the transition is second order, in the universality class of the $3 d$ Ising model [4]. In the right panel of Fig. 4 we show the spatial size dependence of the Binder cumulant $B_{4}$ of the magnetization-like observable, which is used to fit the value of $\lambda$ at the critical point. The value of the Higgs mass $M_{h}$ at this point, in units of the $W$ mass, marks the critical value for which a second order transition can be obtained. In the absence of a $|\phi|^{6}$ term in the potential, this mass is found to be $67.5(5) \mathrm{GeV}$, in good agreement with [8].
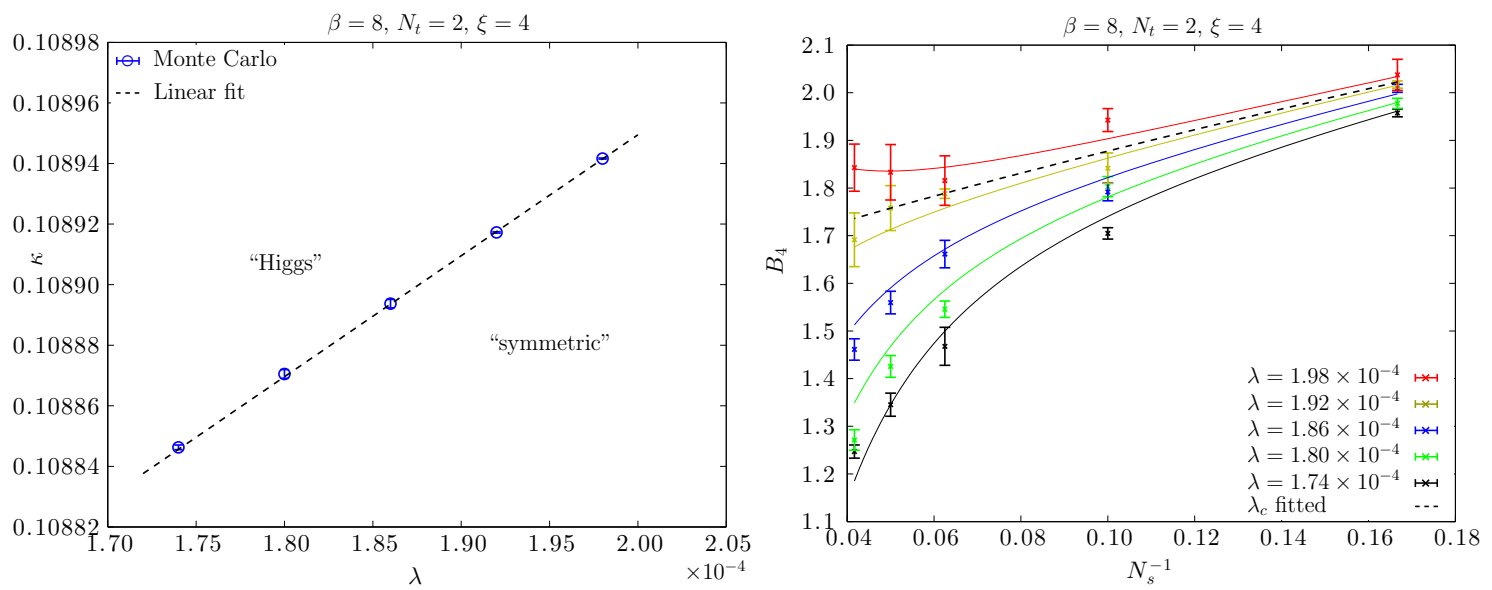

Figure 4: Left panel: The phase diagram at $\lambda_{6}=0$. For small values of $\lambda$ the transition is first order and for large values it is crossover. Right panel: The Binder cumulant $B_{4}$ at the pseudo-critical point as a function of the spatial box size $L$ for different values of $\lambda$. We fit this data to find $\lambda_{c}$ where $B_{4}$ takes the value of the $3 d$ Ising model universality class in the thermodynamic limit.

We now turn on the $|\phi|^{6}$ operator by reweighing the ensembles generated without it. Since the location of the critical point depends on $\lambda_{6} \propto M_{\mathrm{BSM}}^{-2}$, the coefficient of $|\phi|^{6}$, a simultaneous reweighing in $\kappa, \lambda$ and $\lambda_{6}$ is needed. In the left panel of Fig. 5 we show $B_{4}$ in the case where $\lambda_{6}=10^{-7}$. The critical $\lambda$ decreases with $\lambda_{6}$ but due to the positive curvature generated by the $|\phi|^{6}$ term the Higgs mass increases with $\lambda_{6}$. From the linear response of $M_{h}$ to $\lambda_{6}$ we can determine the trajectory of the tricritical point in the $\left(M_{h}, M_{\mathrm{BSM}}\right)$-plane, which is shown in the right panel of Fig. 5. This should be compared with the left panel in Fig. 3: we see that the two models yield consistent results and that they are completely consistent with perturbation theory [11].

\section{Conclusions}

We have investigated two simplified versions of the Standard Model, the Higgs-Yukawa model and a gauge-Higgs model, in the presence of a higher dimension operator, $|\phi|^{6}$, parametrically 

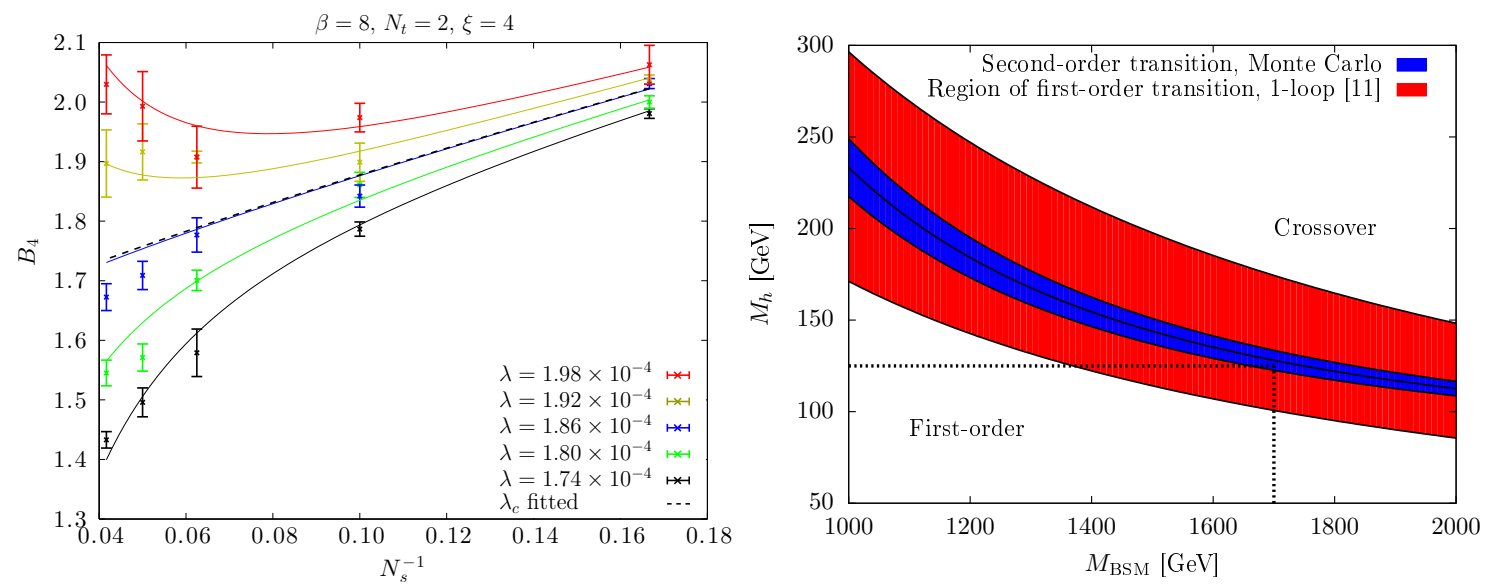

Figure 5: Left panel: The same as the right panel of Fig. 4 but for $\lambda_{6}=10^{-7}$. Right panel: The tricritical line in the $\left(M_{\mathrm{BSM}}, M_{h}\right)$-plane obtained by extrapolating the linear response from small $\lambda_{6}=M_{\mathrm{BSM}}^{-2}$. Above the central line in the blue region the transition is a crossover and below it is first order. The red region is obtained from a tree-level evaluation of the Higgs potential with an additional one-loop thermal mass [11].

suppressed by two powers of a "new physics" energy scale, labeled $M_{\mathrm{BSM}}$. We have determined the curve in the $\left(M_{h}, M_{\mathrm{BSM}}\right)$-plane ( $M_{h}$ being the Higgs mass) where the electroweak finite temperature transition turns first order. This is relevant in the context of electroweak baryogenesis where a strong first order transition is needed in order to fulfill all Sakharov conditions. For the experimental value of the Higgs mass, $M_{h}=125 \mathrm{GeV}$, both models require $M_{\mathrm{BSM}}$ to be around $1.5 \mathrm{TeV}$, which is well within what will be probed by run II of LHC. The fact that both models yield similar results indicates that the Higgs potential itself is most important when determining $M_{\mathrm{BSM}}$, whereas the gauge and fermion degrees of freedom yield only small corrections, captured well by perturbation theory.

\section{References}

[1] J. R. Espinosa and M. Quiros, Phys. Lett. B 353 (1995) 257 [hep-ph/9504241].

[2] V. Branchina, E. Messina and M. Sher, Phys. Rev. D 91 (2015) 013003 [arXiv:1408.5302 [hep-ph]].

[3] B. Grzadkowski, M. Iskrzynski, M. Misiak and J. Rosiek, JHEP 1010 (2010) 085 [arXiv:1008.4884 [hep-ph]].

[4] K. Rummukainen, M. Tsypin, K. Kajantie, M. Laine and M. E. Shaposhnikov, Nucl. Phys. B 532 (1998) 283 [hep-lat/9805013].

[5] O. Akerlund and P. de Forcrand, arXiv:1508.07959 [hep-lat].

[6] D. Y.-J. Chu, K. Jansen, B. Knippschild, C.-J. D. Lin and A. Nagy, Phys. Lett. B 744 (2015) 146 [arXiv:1501.05440 [hep-lat]].

[7] P. Hegde, K. Jansen, C. J. D. Lin, and A. Nagy, "Proceedings, 31st International Symposium on Lattice Field Theory (Lattice 2013)”, PoS LATTICE2013, 058 (2014), arXiv:1310.6260 [hep-lat].

[8] F. Csikor, Z. Fodor and J. Heitger, Phys. Rev. Lett. 82 (1999) 21 [hep-ph/9809291].

[9] J. Steinbauer, "Lattice investigations of the Electroweak phase transition in the Standard Model and beyond", Master's thesis, ETH Zurich, Zurich, Switzerland (2015).

[10] J. Steinbauer and P. de Forcrand, In preparation.

[11] C. Grojean, G. Servant and J. D. Wells, Phys. Rev. D 71 (2005) 036001 [hep-ph/0407019]. 